\title{
SOME OBSERVATIONS ON LEPROSY IN GREAT BRITAIN AT THE PRESENT TIME.
}

\author{
BY J. M. H. MACLEOD, M.D., F.R.C.P. \\ Consulting Physician for Diseases of the Skin for Charing Cross Hospital and St. John's \\ Hospital for Skin Diseases. \\ Consulting Physician for St. Giles' Homes for British Lepers.
}

IN 1909 the Second International Congress on Leprosy was held at Bergen and passed the following resolution :-

"Leprosy is a disease which is contagious from person to person, whatever may be the method by which this contagion is effected. Every country, in whatever latitude it may be situated, is within the range of possible infection by leprosy, and may, therefore, usefully undertake measures to protect itself."

The lapse of more than twenty years has only borne out the wisdom of this resolution. Up to that time there was a feeling in this country that leprosy was not contagious and that any method of enforced segregation was as unnecessary as it was cruel. This fallacious doctrine of non-contagiosity has died a slow death in the medical mind, and now all of us who have had occasion to deal with leprosy must know how erroneous and misleading it was. Many of us must be cognizant with cases where men have gone from this country to live abroad in some district where leprosy was rife, and through close association with it, become infected. In 1925 I reported a series of four contact cases of leprosy contracted in this country, three of them in persons born here and who had never been abroad. In these cases there was a history of prolonged intimate association and actual contact with some other member of the family who suffered from the disease but had contracted it abroad (Brit. Med. Journ., 1925, i, p. 107).

At the present time we have an indefinite number of cases of leprosy in Great Britain. With the exception of the above-mentioned contact cases, they are usually in British subjects, who have come from abroad to settle here either with the knowledge and obvious signs of their affliction or in the incubation period without evidences of it and with the symptoms appearing later.

The exact number of the cases it is impossible to estimate, and such numbers as from 75 to 10o, which are usually given, though possibly not far out, are simply guesswork.

Several reasons are responsible for our want of precise knowledge with regard to the number of cases. First they are liable to be hidden away and the existence of the disease carefully concealed both by the patient and the relatives; then they are apt to go unrecognized, as the disease is fortunately so comparatively rare in this country, that only the few medical men who have been in practice abroad, are capable of diagnosing it ; and most important of all, because it has not been made notifiable in this country.

So long as the cases are in a quiescent phase, especially nerve cases, there seems little risk of infection from them, and there is no urgent need for their isolation, but in active cutaneous cases with ulcerating sores, and the nasal and buccal mucosa teeming with bacilli, it is quite another matter and some form of control and supervision is essential 
for the public safety. At the present time the housing of cases of leprosy in this country is a matter of concern to all who have to deal with them. Advanced cases are liable to be helpless cases and too often to be neglected by their relatives at home. Lodging houses naturally refuse to take them if they are aware of it. The general hospitals only in rare instances will admit them, and if they do, the excitement and panic liable to arise among the nurses and the other patients generally leads to their rapid discharge. Their only remaining shelter is the municipal or county infirmary of the town or district from which they come, where they are regarded as unwelcome guests. Should cases become mental, the difficulties of housing are further increased, as every obstacle is put in the way of their admission to a mental asylum, owing to the excessive cost of providing special attendance and the segregation which is required.

It was all those difficulties which led, in I9I3, to the foundation of the St. Giles' Homes for British Lepers, a charity which is entirely supported by voluntary contributions and in no way subsidized by the State.

These Homes provide accommodation for twelve male and two female patients, and are usually full. They are situated in the depth of the country some fifty miles from London. The nursing and attendance are done by a community of Anglican Franciscan nuns under a Mother Superior, thus eliminating the great nursing problem, for lay nurses, if they can be got to undertake this work, though there is little or no risk of their being infected, are liable sooner or later to succumb to the strain of it and require to be relieved.

The medical attendance at the Homes is carried out by a local doctor and a visiting leprologist of wide experience. At these Homes I have had the opportunity during the last ten years of appreciating to the full the problems connected with its management and treatment.

Leprosy is a disease that is full of mysteries and difficulties. There is no explanation at present as to why in one group of cases the skin is the site of election for the attack and growth of the bacilli, and in another it is the sheaths of the nerves. Though the lepra bacillus occurs in vast profusion in nodules and other leprotic lesions and its demonstration by the microscope is simple, no one has so far succeeded in cultivating it, nor have any of the lower animals been found to be susceptible to it. Experimental inoculations from man to man have given doubtful or negative results. The length of the incubation period is unknown, the mode of transmission is uncertain, and a reliable specific remedy for it, comparable to mercury in syphilis, has yet to be discovered.

From time to time workers in various laboratories, such as Kedrowski, Bayon and others, have announced their success in growing on some medium or other acid-fast bacilli which they believed to be the Bacillus lepra, but when their experiments have been repeated independently, using identical media and under as near as possible the same conditions, negative results have invariably followed. This has interfered with the preparation of a satisfactory vaccine, and in any attempts which have been made to treat leprosy by vaccines some such method has had to be adopted as pounding down a leprotic nodule in normal saline, or raising a blister over a nodule by freezing with $\mathrm{CO}_{2}$ and obtaining bacilli from the fluid contents.

For some time it was thought that an animal susceptible to leprosy had been found in the rat and that the affection known as rat leprosy, which is also due to an acid-fast bacillus, was related to human leprosy. This, however, was proved to be incorrect, and 
it has been found that so-called "rat leprosy" is a highly contagious disease among rats and quite distinct from human leprosy.

With regard to its mode of transmission there is still great diversity of opinion. In this country the influence of Hutchinson still lives in the tenacity with which the "fish theory" of its ætiology has persisted in spite of the facts that leprosy can occur in districts where fish is unobtainable, and that no one has ever found anything even remotely resembling a lepra bacillus in decayed fish, nor succeeded in preparing from fish a medium in which the bacillus will grow.

Of the other articles of diet which have been incriminated, such as rice, maize, fruits, \&c., none has attracted so much attention as fish.

Perhaps the most prevalent view is that it is transmitted by biting insects such as mosquitoes, flies, fleas, ticks and bugs. In this connection experiments have been carried out by feeding insects on leprous sores and fluids and examining the probosces and the alimentary tract for bacilli some hours later, and certain of them have given positive results. Of these insects the bug has been regarded as the most likely carrier, but so far without actual proof.

Of all the problems connected with leprosy the one which is attracting most attention at the moment is treatment, and at the St. Giles' Homes we have had a fairly good opportunity of estimating the relative value of the various forms of therapy at present in use. For although the actual number of cases there is comparatively small, these cases have been carefully watched over a long period, and thus the lessons to be derived from them may compare favourably: with those from observations elsewhere of larger numbers of cases where the possibility of studying the individual case and following up its after history may be more difficult and may vary inversely with their number.

The estimation of the value of treatment in leprosy is beset with difficulties, owing to the seemingly spontaneous periods of quiescence and activity which occur in its course apart from treatment, the influences of environment, suitable food, season and climate, and its self-curative tendency. Periods of remission, while the patient is under some form of treatment, naturally advertise the remedy in use, and may endow it with curative powers which are fictitious.

Nor do we find it easy to say when a case is cured, for even though no sign of activity of the disease may be present, and the patient may have been free from bacilli for a varying time, recrudescence may sooner or later take place. It is more in keeping with the general experience to classify such cases as arrested rather than cured. Leprosy has long been recognized to have a tendency to be self-curative, or so-to-speak, to " burn out," but only after long years of suffering, leaving a wreck of humanity, deformed, mutilated, probably blind and crippled by disabilities so great as to make life scarcely worth living.

The treatment of leprosy may be conveniently divided into general, local and special.

\section{General Treatment.}

So far as we have found at the Homes, where the majority of cases are of the advanced type, and only the few in an early stage when treatment might be expected to cause an arrest, the benefit that has been obtained from the general régime has been greater than that which could have been attributed to any form of special therapy. As 
a result of the healthy surroundings, fresh air, exercise, good food, mental occupation, recreation and suitable tonic treatment on general medical principles, the resistance of the patients is increased, and an all-round improvement results. This, of course, is specially noticeable in recently admitted cases. Climate and season too have their influence, and there is usually a marked improvement in the cases in the warm dry weather of summer and a tendency to replace in the cold and damp of the winter months.

\section{Local Treatment.}

The local treatment is symptomatic and palliative. It is very necessary for the relief of discomfort or pain, but has little influence on the progress of the disease. It demands skill, knowledge and resource. Ulcers have to be treated, possibly excised and grafted, dead bone or useless members may have to be removed surgicaliy, and all manner of eye symptoms may have to be combated. We have made attempts to hasten cure by the destruction of nodules and plaques by various methods such as cauterization, the application of trichloracetic acid, subcutaneous injections of carbolic acid solutions, intradermal injections of esters of hydnocarpus, chaulmoogra oil, \&c., and their derivatives, and in some instances succeeded in causing the complete involution or healing of the local lesions to the relief and comfort of the patient. Such beneficial results, however, have no real influence on the course of the disease, but they are of great value from a psychological point of view. Patients with leprosy are apt to become despondent to the detriment of their general health, and any form of systematic local treatment followed by even a minimum of benefit may assist in temporarily dispelling the prevalent gloom.

\section{Special Treatment.}

It will be noted here that the adjective special has been substituted for specific, for at the present time it cannot be said that a specific remedy for leprosy, corresponding to mercury or salvarsan in syphilis, has been discovered.

The fact that the list of so-called specifics is so long and varied is in itself an index of their uncertainty. They vary from metallic preparations and aniline dyes to vaccines and vegetable oils and their derivatives, and their number is legion. Of the inorganic preparations, the two which have attracted most attention lately have been antimony and iodide of potassium.

Antimony can be given by intramuscular injection in the form of the tartar emetic ( $\mathrm{I}$ gr. in Io c.c. of normal saline solution) or of colloidal antimony in doses of 2 to 6 c.c. The colloidal injections are preferable, as they produce less local irritation and have no toxic effect.

Iodide of potassium has been advocated as a means of causing the breaking up and flattening of the nodules as it does the gummata in syphilis. Though it may sometimes be of benefit it is uncertain, as the disintegration of tissue and bacilli caused by it is associated with a toxic febrile reaction which, if excessive, may be harmful, especially in advanced cases, and it has also the disadvantage of being a depressant.

Aniline Preparations. - The aniline dye recommended is mercurochrome (soluble 220). It is given in doses of 3 to 5 c.c. of a I per cent. solution in distilled water injected intravenously once a week. Its chief value is believed to be its power of clearing up concomitant septic conditions such as ulcers, pyorrhœea, \&c., and of 
reducing leprotic fever. Intradermally injected it is said to cause liquefaction and abscess formation of nodules. This remedy is still in its experimental stage.

Vaccines.-Both specific vaccines prepared from the $B$. leprae and non-specific vaccines have been employed.

The specific vaccines have been emulsions made from excised lepromata, serum squeezed from excoriated nodules and laden with bacilli, or the contents of artificially produced blisters. Several of the cases have been so treated. In one case an apparent arrest was obtained by this means, and for a period of about four years all signs of the disease disappeared, but recently there has been a relapse, with the development of iridocyclitis, defective vision and a perforating ulcer. Another case which has been under this treatment from time to time during the last five years has not benefited from it.

By means of non-specific vaccines and the production of protein shock, more dramatic results have been achieved. Of the various methods of producing this, the simplest way is by typhoid vaccines and the intravenous injection of T.A.B., commencing with 50 millions, and repeating at weekly intervals. The effect of such treatment may be to produce a remarkable improvement, especially in cutaneous cases, where the nodules become flattened out, ulcerations become dried up and heal, and the general condition is ameliorated. Unfortunately this improvement is liable to be temporary, but in suitable cases it is well worth while. It must be used with caution, as in quiescent cases it may do harm, from causing the breaking up of the glutinous substance, which holds the bacilli together, allowing an embolic shower of bacilli to reach the circulation and so causing a generalization of the disease.

Tuberculin injections which also give rise to a marked non-specific reaction in leprosy should not be employed, as they are apt to light up latent foci of tuberculosis which may be concomitant with the leprosy.

Vegetable Oils.-The use of chaulmoogra oil in leprosy is centuries old, and like cod-liver oil in tuberculosis, has been regarded as a sheet-anchor in the treatment. It has been used externally and internally, by inunction, injection and ingestion and even at the present time one is almost tempted to aver that the cases which do best are those which can digest the largest quantity of this oil. Efforts have been made to reduce its nauseating effects by keeping the patient on a milk diet, or combining the oil with malt, but these have been disappointing.

This difficulty has been largely overcome by the use of derivatives of the allied hydnocarpus oil, which are capable of being injected intramuscularly and intravenously. Of these one of the most popular at the moment is "alepol," which when combined with I per cent. novocain can be injected painlessly.

Though specific properties are attributed to alepol and such preparations and their injection is occasionally associated with febrile reactions, we have yet to be convinced that they are more beneficial than chaulmoogra oil itself, and in no case in our limited experience at the Homes has a cure been effected by their use.

Recently the method of intradermal infiltration of the lesions themselves by hydnocarpus preparations is being given a trial, and for this purpose the iodized ethyl esters are more convenient than the oil itself, as they infiltrate more easily. A series of injections are given once or twice a week of about half a minim each till a dose of $0^{\circ} 5$ to 3 c.c. has been reached. By this means the nodules injected have become flattened. The ultimate result of the treatment is being watched with interest. 
It must be remembered that leprosy like tuberculosis is a bacillary disease, and unlike spirochætal diseases such as syphilis and yaws, does not readily respond to drugs. As in tuberculosis, so in leprosy, in the present state of our knowledge, we must depend as much if not more on segregation and a sanatorium régime to increase the resistance of the patient or on the use of protein shock in suitable cases than on any form of so-called specific therapy which has yet been introduced.

\section{ROUND THE WARDS WITH MR. LOCKHART-MUMMERY AT ST. MARK'S HOSPITAL.}

St. Mark's Hospital, in the City Road, London, is unequalled in the facilities it affords for studying a large number of cases of one particular region collected in a single building, and in charge of a staff of men who practise almost exclusively in this one particular branch of surgery. The Hospital contains seventy-five beds, and last year over I, roo operations were performed, all for conditions of the rectum or colon. There is at present no other institution in the world which affords similar opportunities of seeing many different kinds of disease, and abnormal conditions, of the large bowel. As regards equipment this Hospital is thoroughly up-to-date, and cases can be seen to the best advantage by anyone visiting the clinics.

\section{Case of Epithelioma of Anus Treated by Radium.}

The first case shown was that of a man, aged 55, who had originally come to the Hospital with an epithelioma of the anal margin. The case had been treated by radium nine months previously. The original tumour was about $\mathrm{I} \frac{1}{2}$ in. in diameter and extended out on to the skin of the perineum in front of the anus and up the anal canal for about an inch. Microscopic slides of the specimen removed for examination were demonstrated showing the typical appearance of squamous carcinoma. There were no enlarged glands. The case was treated with radon seeds and radium needles. Radium equivalent to $285 \mathrm{mg}$. was used and the total dose was about 2,500 mg. hours. The epitheliomatous ulcer began to heal in ten days and had entirely healed over in three and a half weeks. At the present time, nine months later, there is no sign of anything wrong. The anal opening is normal and there is no detectable scar.

It was pointed out that squamous-celled carcinoma could be very successfully treated with radium, and that if, as appeared to be the case in this instance, the correct dose was used the results were little short of miraculous. Unfortunately there was at the present time no accurate means of determining the proper dose, and the experience of the surgeon in similar cases was the only guide. It was pointed out that we are still quite in the dark as to the way in which radium acts upon the tumour. It was clearly not merely a question of the destruction of the cancer cells, as no sloughing occurred, the cancerous ulcer simply healed up, leaving normal tissue in its place. If, on the other hand, an overdose of radium was used either sloughing occurred or dense fibrous tissue was formed. But when the dose was correct the tissues simply resumed a normal appearance in the course of a few weeks without any violent reaction and with the minimum of scar tissue. 